\title{
A PROPERTY OF TRANSFERABLE LATTICES
}

\author{
G. GRÄTZER ${ }^{1}$
}

\begin{abstract}
A lattice $K$ is transferable if whenever $K$ can be embedded into the ideal lattice of a lattice $L$, then $K$ can be embedded in $L$. An element is called doubly reducible if it is both join- and meet-reducible. In this note it is proved that every lattice can be embedded into the ideal lattice of a lattice with no doubly reducible element. It follows from this result that a transferable lattice has no doubly reducible element.
\end{abstract}

1. Introduction. In [5] two concepts of transferability of a lattice were introduced, named transferability and weak transferability in [2] and named sharp transferability and transferability, respectively, in this paper and [4]. A rather satisfying theory of sharp transferability can be found in [2] and [4]; see also [3] for the case of semilattices. Recently K. Baker proved that all finite projective lattices are transferable (see [1]). Still, the only known property of transferable lattices is the one announced in [5] without proof. The purpose of this note is to supply a proof of this property (see Theorem below).

First, two definitions. A lattice $K$ is called weakly transferable iff whenever $K$ can be embedded into the lattice of all ideals of a lattice $L$, then $K$ can also be embedded into $L$. Observe, that in the papers referred to above the finiteness of $K$ is also assumed; for the purposes of this note, however, it is not necessary to assume that $K$ is finite. An element $a$ of the lattice $K$ is doubly reducible iff there exist elements $x, y, z, u$ of $K$, all distinct from $a$, such that $a=x \vee y=z \wedge u$.

THEOREM. A transferable lattice contains no doubly reducible element.

2. Proof of the Theorem. Let $A$ and $B$ be posets. The lexicographic product of $A$ and $B$, denoted by $A \otimes B$, is a poset defined on $A \times B$ with the ordering $\left(a, a^{\prime} \in A, b, b^{\prime} \in B\right)$ :

$$
\langle a, b\rangle \leqq\left\langle a^{\prime}, b^{\prime}\right\rangle \text { iff } a<a^{\prime} \text { or } a=a^{\prime} \text { and } b \leqq b^{\prime} .
$$

In this note, let $A$ and $B$ be lattices and let $B$ have a least element 0 and largest element 1 .

Received by the editors July $16,1973$.

AMS (MOS) subject classifications (1970). Primary 06A20; Secondary $02 \mathrm{H} 05$.

Key words and phrases. Lattice, transferable, doubly reducible, ideal lattice.

${ }^{1}$ Research supported by the National Research Council of Canada. 
Lemma 1. $A \otimes B$ is a lattice and, for $a, a^{\prime} \in A, b, b^{\prime} \in B$, we have that

$$
\langle a, b\rangle \vee\left\langle a^{\prime}, b^{\prime}\right\rangle=\left\langle a \vee a^{\prime}, b^{\prime \prime}\right\rangle
$$

with suitable $b^{\prime \prime} \in B$; in fact, if $a$ and $a^{\prime}$ are incomparable, then $b^{\prime \prime}=0$.

Proof. Trivial; observe, that if $a<a^{\prime}$, then $b^{\prime \prime}=b^{\prime}$; if $a^{\prime}<a$, then $b^{\prime \prime}=b ;$ if $a=a^{\prime}$, then $b^{\prime \prime}=b \vee b^{\prime}$.

Call an element $p$ join-reducible if $p=x \vee y$ with $p \neq x$ and $p \neq y$; otherwise $p$ is join-irreducible. The dual concepts are meet-reducible and meetirreducible. From Lemma 1 we conclude immediately:

Corollary 2. All join-reducible elements of $A \otimes B$ are of the form $\langle a, b\rangle$ where $b=0$ or $b$ is join-reducible in $B$.

Corollary 2 and its dual yield:

COROLlaRY 3. Let us assume that $B$ has more than one element, and 0 is meet-irreducible, and 1 is join-irreducible in $B$. Then all doubly reducible elements of $A \otimes B$ have the form $\langle a, b\rangle$, where $b$ is doubly reducible in $B$. In particular, if $B$ has no doubly reducible element, then neither does $A \otimes B$.

Now we map ideals of $A$ into ideals of $A \otimes B$. Let $I$ be an ideal of $A$. We set

$$
I=\{\langle a, b\rangle \mid a \in I, b \in B\} .
$$

Lemma 4. For any ideal I of $A$, the set $I$ is an ideal of $A \otimes B$. The map $I \rightarrow I$ is one-to-one, and for ideals $I$ and $J$ of $A$ it satisfies

$$
I \wedge J=(I \wedge J)^{-}
$$

it also satisfies

$$
I \vee J=(I \vee J)^{-},
$$

provided that $I \vee J$ is not a principal ideal:

Proof. It follows immediately from (1) and (2) that (3) defines an ideal. Now, $\langle a, b\rangle \in \bar{I} \wedge \bar{J}$ iff $\langle a, b\rangle \in \bar{I}$ and $\langle a, b\rangle \in \bar{J}$, which is, by (3), equivalent to $a \in I$ and $a \in J$, that is, to $a \in I \wedge J$, which means that

proving (4).

$$
\langle a, b\rangle \in(I \wedge J)^{-},
$$

By (4), the inclusion " $\subseteq$ " is obvious in (5). To prove the reverse inclusion, let

$$
\langle a, b\rangle \in(I \vee J)^{-} \text {. }
$$


Then $a \in I \vee J$ by (3); since, by hypothesis, $I \vee J$ is not principal, there exists an $a^{\prime} \in I \vee J$ satisfying $a<a^{\prime}$. Also, since $a^{\prime} \in I \vee J$, we get elements $i, j$ of $A$ with $a^{\prime} \leqq i \vee j, i \in I, j \in J$. By (3), $\langle i, 0\rangle \in I,\langle j, 0\rangle \in \bar{J}$, and so, using (1) and (2),

$$
\langle a, b\rangle\langle\langle i \vee j, 0\rangle=\langle i, 0\rangle \vee\langle j, 0\rangle \in I \vee \bar{J},
$$

proving the reverse inclusion, and thus Lemma 4.

Next, we need a trivial construction.

Lemma 5. Let $K$ be an arbitrary lattice. $K$ has an embedding $\varphi$ into the lattice of all ideals of a suitable lattice $L$ such that, for all $a \in K, a \varphi$ is a nonprincipal ideal of $L$.

Proof. For instance, let $N$ be the chain of natural numbers, $L=K \times N$, and, for $a \in K$, set $a \varphi=\{\langle x, n\rangle \mid x \leqq a\}$.

Combining the embeddings of Lemma 4 (with, say, $B$ the two-element chain) and Lemma 5 we obtain the main result of this note:

THEOREM 6. Every lattice $K$ can be embedded into the lattice of all ideals of some lattice $L$ with no doubly reducible element.

The Theorem of the Introduction follows immediately from Theorem 6. Indeed, if $K$ is a transferable lattice, then we embed $K$ into $I(L)$ by Theorem 6 , where $L$ is a lattice with no doubly reducible element. By transferability, $K$ can be embedded into $L$; hence $K$ has no doubly reducible element.

\section{REFERENCES}

1. K. Baker and A. Hales, From a lattice to its ideal lattice, Algebra Universalis (to appear).

2. H. S. Gaskill, Transferability in lattices and semilattices, Ph.D. Thesis, Simon Fraser University, 1972.

3. - On transferable semilattices, Algebra Universalis 2 (1972), 303-316.

4. H. Gaskill and C. R. Platt, Transferable lattices (manuscript).

5. G. Grätzer, Universal algebra, Trends in Lattice Theory (Sympos., U.S. Naval Academy, Annapolis, Md., 1966), Van Nostrand Reinhold, New York, 1970, pp. 173210. MR 43 \#7389.

Department of Mathematics, University of Manitoba, Winnipeg R3T 2N2, Manitoba, Canada 